Thorax, 1978, 33, 803-805

\title{
Diagnosis of a thoracic inlet tumour by transbronchial biopsy
}

\author{
DAVID S PRATT \\ From the Veterans Administration Hospital, Denver, Colorado 80220, USA
}

\begin{abstract}
Pratt, D S (1978). Thorax, 33, 803-805. Diagnosis of a thoracic inlet tumour by transbronchial biopsy. A 67-year-old man presented to the pulmonary service with left shoulder and arm pain. Examination disclosed a left Horner's syndrome. Supraclavicular exploration at another hospital was not helpful. Transbronchial biopsy through a flexible fibreoptic bronchoscope retrieved tissuc showing poorly differentiated carcinoma. There are no previous reports of the use of the technique in the diagnosis of a Pancoast tumour with a normal standard chest radiograph.
\end{abstract}

Thoracic inlet tumours affecting motor, sensory, and autonomic nerves may be difficult to see on routine chest radiographs (Hepper et al, 1966). Despite the symptoms of severe shoulder and arm pain, physical findings, except for Horner's syndrome, are often absent. Additional laboratory studies seldom help.

Although the most common cause of Pancoast syndrome is bronchogenic carcinoma with contiguous spread (Paulson, 1975), other malignant conditions can cause these findings, including Hodgkin's disease, laryngeal carcinoma, sarcoma, and metastatic carcinomas. Non-malignant disease, such as tuberculosis, has also been reported as causing Pancoast syndrome (Vamos and Papp, 1960; Camelot et al, 1964). The high likelihood of malignancy often persuades physicians to treat all patients with Pancoast syndrome as if the cause were malignant (Hepper et al, 1966). Radiation therapy to a non-malignant lesion would obviously be a serious error and therefore many would agree with McGoon (1964) and Paulson (1975) that histological diagnosis is important in managing patients with Pancoast syndrome. Diagnosis of the aetiology of Pancoast syndrome can be achieved by formal axillary thorocotomy, supraclavicular exploration, or needle aspiration "biopsy." Another technique, fibreoptic bronchoscopy, may be useful in establishing the diagnosis.

\section{Case report}

A 67-year-old white male retired office worker sought medical attention because of a one-month history of left shoulder pain. Chest and shoulder radiographs were interpreted as normal, and the pain was treated with analgesics. The pain persisted in his shoulder, and six months later he noted pain and weakness in his left arm. He was once again examined by a physician and was admitted to another hospital. An extensive investigation, which included chest radiographs, bone, liver, and spleen scans, and other laboratory studies failed to establish a diagnosis. Because a neurologist suspected a Horner's syndrome, supraclavicular exploration was done. The subclavian artery was not mobilised; nevertheless, lymph nodes in the area were normal and no obvious tumour was seen. His physicians transferred the patient to the University of Colorado Medical Centre for further investigation. The patient had lost $7 \mathrm{~kg}$ during his illness. He was pale, slender, and in substantial distress from left arm and shoulder pain. The physical examination disclosed a left Horner's syndrome and severe pain in the shoulder on passive movement of the arm. Neurological examination of the left arm suggested motor and sensory loss affecting the eighth cervical and first two thoracic nerve roots.

Chest radiographs were repeated and again interpreted as normal except for a suggestion of scarring in the apical-medial portion of the left lung and hyperinflation (fig 1). Tomograms at 12 and $13 \mathrm{~cm}$ disclosed a definite area of apical density that was asymmetrical when right and left sides were compared (fig 2). Bone scans and shoulder and apical lordotic radiographs were normal. Because of the strong suspicion that the patient had a thoracic inlet tumour, a flexible fibreoptic bronchoscopy was performed. The left apicoposterior 


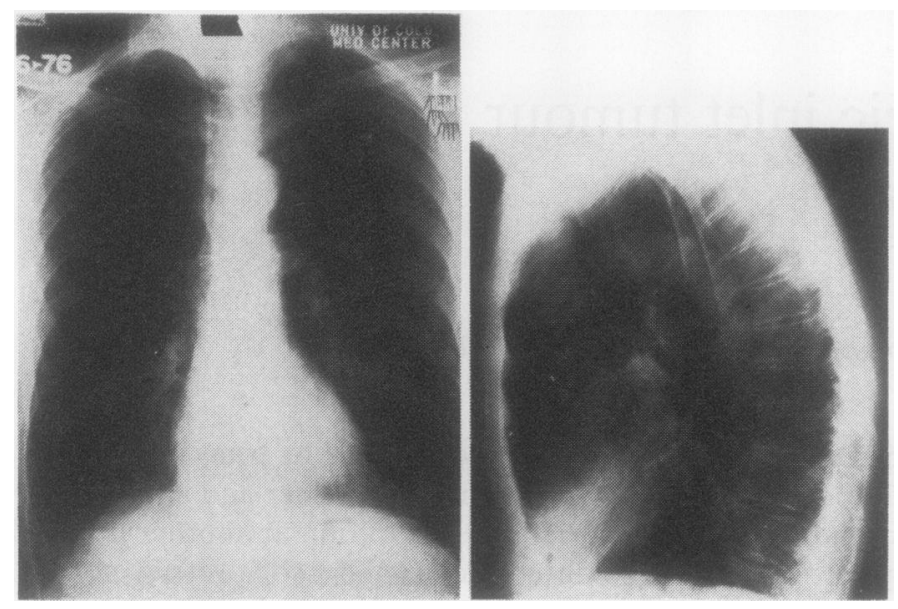

Fig 1 Standard chest radiographs take'n at admission showing left apical "scarring.",

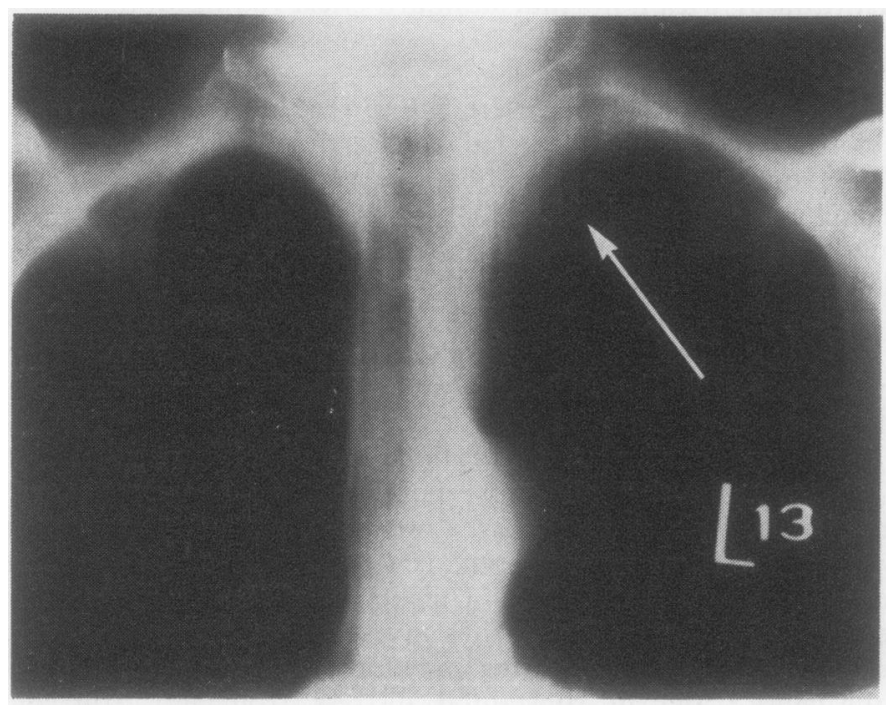

Fig 2 Tomographic cut at $13 \mathrm{~cm}$ showing definite a;ymetry between right and left apices.

bronchus was entered, and under fluoroscopic guidance biopsy forceps were advanced into the area of suspected malignancy. Five biopsy specimens were taken and fixed in formalin. The tissue was friable and anthracotic, but its removal prompted little bleeding. The patient had no complications from the procedure.

Histological examination showed poorly differentiated carcinoma (fig 3). The patient was irradiated with 3000 rads to his left apex and three weeks later had an en bloc dissection of his left upper lobe. At operation, the tumour was adherent to ribs, vertebral bodies, nerves, and vessels. The heads of the second and third ribs were removed as well as the transverse processes of the first two thoracic vertebrae. The tumour was removed along with the left upper lobe. After a difficult four-month hospital recuperation the patient was discharged home. He died three months later of bacterial pneumonia at another hospital.

\section{Discussion}

Since its introduction into America in 1969, the flexible fibreoptic bronchoscope has had a considerable impact on diagnosis in chest medicine. The periphery of the upper lobe has always been an inaccessible area to rigid bronchoscopy. This limitation is serious, considering the frequency of upper lobe malignancy (Watson, 1968). The flexible instrument allows deep penetration into the upper lobes to the fourth or fifth order bronchi (Kovnat 


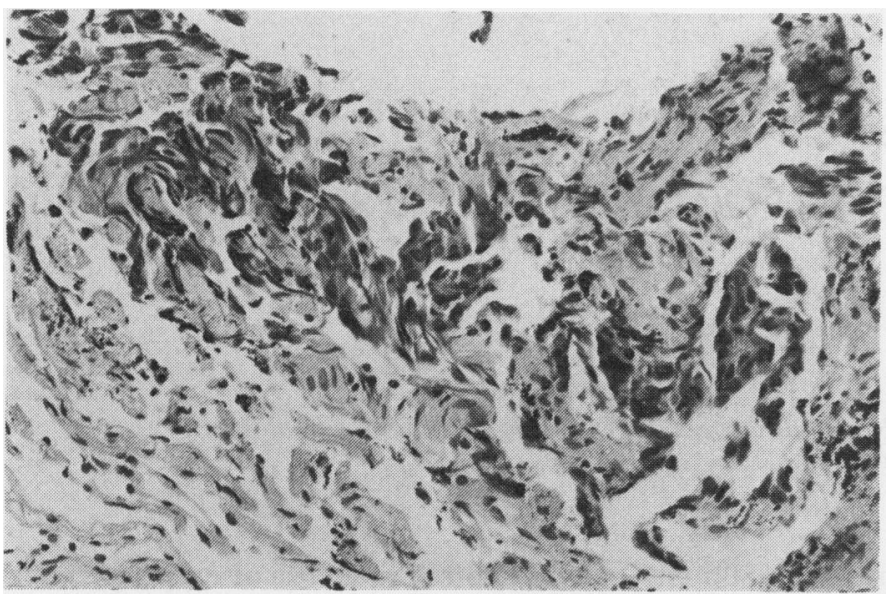

Fig 3 Malignant cells retrieved from transbronchial biopsy (Haematoxylin and eosin $\times 242$ ).

et al, 1974). Biopsy forceps can be advanced to the distal parenchyma and even to the pleural surface.

The usual approach to thoracic inlet tumour diagnosis is via thoracotomy (McGoon, 1964). This has a substantial attendant morbidity and mortality, especially if a lobectomy is undertaken at the same time (Glenn, 1975). Deep transcervical dissection has been recommended by McGoon (1964). That operation has a definite morbidity, but certainly less than formal thoracotomy. Walls et al (1974) have recommended needle aspiration, which can give a high yield of positive results. Their method requires skill in the hands of the operator and expertise in cytopathology. Pneumothorax occurs in about $10 \%$ of needle aspiration biopsies.

The technique described in this report has many advantages over the above methods. The bronchoscopy is done with local anaesthesia. It is performed with fluoroscopic guidance and does not require an operating room or more than one assistant. The hospital stay is seldom more than 24 hours. Complication rates are low (Ellis, 1975), especially in diseases where the adherent pleura protects the patient from pneumothorax.

Transbronchial biopsy has a high benefit : risk ratio, low morbidity, and low cost (both direct and indirect). Paulson (1975) has recommended the use of this technique for the diagnosis of tumour in Pancoast syndrome for several years, but has not reported results. This report describes the first time a thoracic inlet tumour has been diagnosed using transbronchial biopsy in the face of a negative plain radiograph. Should transbronchial biopsy fail, the other, more invasive diagnostic techniques remain available.

\section{References}

Camelot, A, Crinquette, J, and Callens, J (1964). Benign tumors of the bronchi causing Pancoast syndrome. Journal des Sciences Medicales de Lille, 82, 719-26.

Ellis, J H (1975). Transbronchial lung biopsy via the fiberoptic bronchoscope. Chest, 68, 524-532.

Glenn, W W L (1975). Thoracic and Cardiovascular Surgery with Related Pathology, pp 358-359. Appleton, Century, Crofts Publishers, New York.

Hepper, N G G, Herskovic, T, Witten, D M, Mulder, D W, and Woolner, L B (1966). Thoracic inlet tumors. Annals of Internal Medicine, 64, 979-988.

Kovnat, D M, Rath, G S, Anderson, W M, Snider, G L (1974). Maximal extent of visualisation of bronchial tree by flexible fiberoptic bronchoscopy. American Review of Respiratory Disease, 110, 8890.

McGoon, D C (1964). Transcervical technique for removal of specimen from superior sulcus tumor for pathologic study. Annals of Surgery, 159, 407410.

Paulson, D L (1975). Carcinomas of the superior pulmonary sulcus. Journal of Thoracic and Cardiovascular Surgery, 70, 1095-1102.

Vamos, G, and Papp, A (1960). Pancoast syndrome and pulmonary tuberculosis. Schweizerische Zeitschrift fur Tuberkulose und Pneumonologie, 17, 423-430.

Walls, W J, Thornbury, J R, and Naylor, B (1974). Pulmonary needle aspiration biopsy in the diagnosis of Pancoast tumors. Radiology, 111, 99-102.

Watson, W L (1968). Lung Cancer, page 378. C V Mosby Company, St Louis, Missouri.

Requests for reprints to: David S Pratt, MD, The Mary Imogene Bassett Hospital, Cooperstown, NY 13326. 